\title{
Influence of crystalline defects on magnetic nanodomains in a rare-earth-free magnetocrystalline anisotropic alloy
}

\author{
Dhanalakshmi Palanisamy, ${ }^{1}$ András Kovács, ${ }^{2}$ Omkar Hegde, ${ }^{1}$ Rafal E. Dunin-Borkowski, ${ }^{2}$ Dierk Raabe, ${ }^{1}$ \\ Tilmann Hickel, ${ }^{1}$ and Baptiste Gault ${ }^{1,3}$ \\ ${ }^{1}$ Max-Planck-Institut für Eisenforschung GmbH, 40237 Düsseldorf, Germany \\ ${ }^{2}$ Ernst Ruska-Centre for Microscopy and Spectroscopy with Electrons, Peter Grünberg Institute, \\ Forschungszentrum Jülich, 52425 Jülich, Germany \\ ${ }^{3}$ Department of Materials, Royal School of Mines, Imperial College London, SW7 2BP London, United Kingdom
}

(Received 15 July 2020; revised 30 March 2021; accepted 27 April 2021; published 2 June 2021)

\begin{abstract}
A complex interplay between magnetic domain structure and crystalline imperfections, here twins, is revealed in a rare-earth-free $\mathrm{MnAl}$ bulk magnet. The magnetic domains are observed to be in the nanometer range for a large part of the magnetic structure and to scale with the number density of twins formed during thermal processing. We explain this phenomenon by a reduction in domain-wall energy at the twinned regions as proven by ab initio calculations. In addition, our atomic-scale analysis reveals that the twin boundaries contain excess $\mathrm{Mn}$ atoms that reduce the local magnetization, serving as an obstacle for domain wall motion. These insights can help guide the strategic design of magnetic materials by controlling the initial phase distribution to tailor the twin density and hence, the distribution of domains.
\end{abstract}

DOI: 10.1103/PhysRevMaterials.5.064403

Magnets are used extensively in electric vehicles and for harvesting energy from wind and steam. Magnetic materials draw their properties from arrangements of magnetic domains and their boundaries, i.e., domain walls, which depend directly on the material's microstructure. A magnetic domain is a unit within a material in which the atomic dipole moments are aligned. Two adjacent domains have an angular displacement and are separated by a domain wall (DW) of finite thickness, across which the dipoles change their orientation gradually [1]. The magnetic behavior of the material depends on the distribution of the domains and how they respond collectively to an applied magnetic field. For example, intrinsic properties such as coercivity and remanence depend on the pinning of DWs at interfaces in the microstructure of the material. Hence, DW propagation depends on the metallurgical state of the alloy, providing unique opportunities to tailor properties towards a specific application by exploiting alloy design, i.e., by adjusting the composition and processing of the alloy to produce a specific microstructure. Key parameters that can be used to control magnetic properties are structural and chemical imperfections, including one-dimensional defects (e.g., dislocations), two-dimensional planar defects (e.g., antiphase boundaries and stacking faults), and grain boundaries (GBs) [2-4].

Published by the American Physical Society under the terms of the Creative Commons Attribution 4.0 International license. Further distribution of this work must maintain attribution to the author(s) and the published article's title, journal citation, and DOI. Open access publication funded by the Max Planck Society.
Twin boundaries (TBs) are planar defects that are often found in $A B$-type $L 1_{0}$-ordered (with $A$ and $B$ occupying alternate atomic layers in a tetragonal lattice) magnetocrystalline anisotropic alloys, such as FePt, FePd, and CoPt. They typically form either as strain relaxation defects during unidirectional thin-film growth [5] or as faults associated with atomic reshuffling during heterophase interface migration $[6,7]$. A correlation between the number density of microtwins and magnetic coercivity has been reported $[4,8]$, and it was proposed that microtwins may act as pinning sites for migrating domain walls (DWs), leading to an increase in coercivity $[4,9]$. However, the underlying physical mechanisms for these two aspects had not yet been unveiled.

In this paper, we illustrate the nature of the interaction between these microtwins and magnetic domains in $L 1_{0}$-ordered anisotropic $\tau \mathrm{MnAl}$ by combining direct magnetic domain structure imaging, structural and compositional measurements with near-atomic resolution. We control the number density of twins by the succession of phase transformations during the alloy's processing history and achieve in this way remarkably small magnetic domain sizes on the order of a few nanometers. With the aid of density-functional theory (DFT), we propose two mechanisms for how the microtwins can change the local magnetic domain structure, finally addressing these longstanding open questions.

The MnAl ( $\tau$-phase) intermetallic compound chosen for this study is, among $L 1_{0}$-ordered magnetocrystalline anisotropic materials, a low-cost, rare-earth-free magnet that has attractive ferromagnetic properties [10]. This binary alloy is akin to Heusler alloys: the individual elements ( $\mathrm{Al}$ and $\mathrm{Mn}$ ) are not ferromagnetic, whereas intermetallic $\mathrm{MnAl}$ arranged on a body-centered tetragonal lattice exhibits ferromagnetic 
(a) BSE image of $\tau$-phase

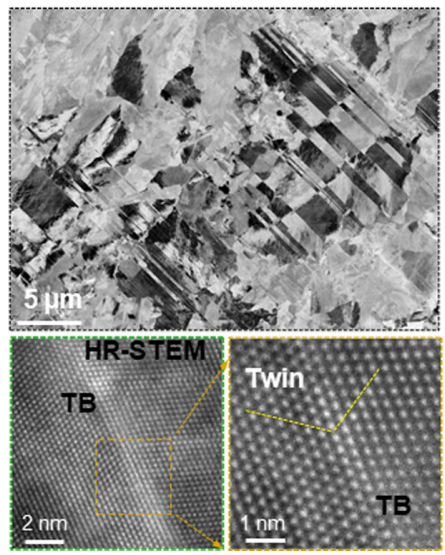

(b)

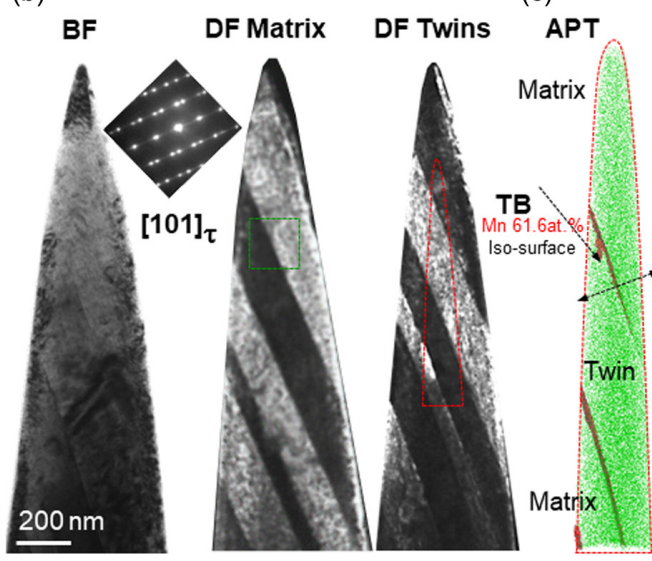

Compositional profile across twin boundary

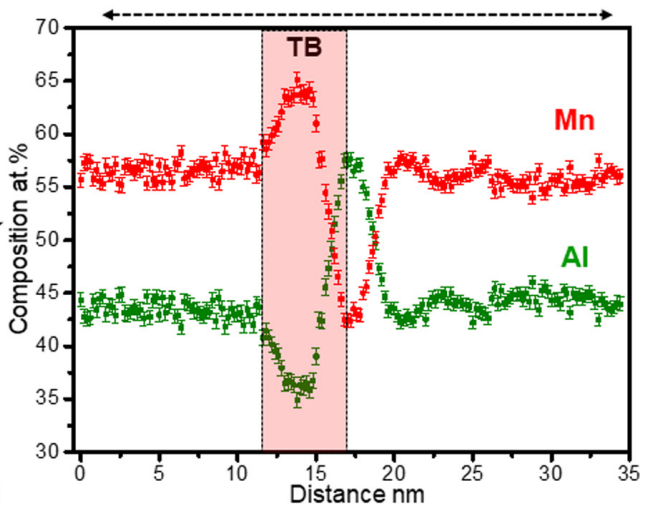

FIG. 1. Magnetic and microstructural analysis of the $\tau$ MnAl alloy. (a) BSE micrograph of a region that contains a high density of microtwins. (b) BF TEM image of an APT specimen recorded close to the $[101]_{\tau}$ zone axis and corresponding DF TEM images showing twinning. High-resolution HAADF STEM images across a TB region taken from the region marked by a green dashed rectangle. The red dashed outline marks the section of the specimen analyzed using APT. (c) APT reconstruction, showing the measured distribution of Al atoms, as well as 61 at.\% Mn isosurfaces that highlight the TBs. Composition profile normal to a TB, revealing confined Mn enrichment up to $\sim 8$ at. $\%$ compared to the matrix.

behavior with a large uniaxial magnetocrystalline anisotropy $\left(\sim 10^{6} \mathrm{~J} / \mathrm{m}^{3}\right)$ along the easy $c$ axis [11]. The ferromagnetic $\tau$ phase nucleates at GBs in the high-temperature hexagonal $\varepsilon$ phase [7,12], with movement of heterophase interfaces resulting in a high density of microtwins in the final microstructure. The coercivity of the alloy can be increased by subsequent nanocrystalline processing up to a value of $\sim 400 \mathrm{kA} / \mathrm{m}$ ( $\sim 5 \mathrm{kOe})[13,14]$, demonstrating that processing can influence magnetic properties.

To this end, an ingot of a MnAl ferromagnetic alloy was melted, followed by homogenization at $1100{ }^{\circ} \mathrm{C}$ for $10 \mathrm{~h}$ and immediate water quenching to retain the high-temperature $\varepsilon$ phase at room temperature. Following heat treatment at $450{ }^{\circ} \mathrm{C}$ for $2 \mathrm{~h}$, the $\varepsilon$ phase had wholly transformed into the ferromagnetic $\tau$ phase $[6,7,15]$. In order to obtain an alloy with a fully transformed $\tau$ phase, the alloy's composition was chosen to be $\mathrm{Mn}_{55} \mathrm{Al}_{45}$ [12]. A magnetic hysteresis loop recorded from the $\tau$-phase alloy reveals a saturation magnetization of $\sim 665.6 \mathrm{kA} / \mathrm{m}$ (Supplemental Material, Fig. S1 [16]). The magnetic hysteresis loop is typical of a ferromagnetic material, with a coercivity of $45 \mathrm{kA} / \mathrm{m}$ (560 Oe).

We first investigated the microstructure of the samples using backscattered electron (BSE) microscopy of numerous microtwins, which correspond to regions of darker and lighter contrast. Structural and compositional analysis of the microtwin regions was performed using correlative transmission electron microscopy (TEM) and atom probe tomography (APT). Figure 1(b) shows a bright-field (BF) TEM image of a needle-shaped specimen for APT recorded close to the $[101]_{\tau}$ zone axis. The corresponding selected area electron diffraction pattern reveals $\{111\}$ twinning diffraction spots alongside primary matrix reflections. The microtwins are formed by the shear of $1 / 6\langle 112\rangle$ partial dislocations on $\{111\}$ atomic planes [7]. The twins have different thicknesses, while maintaining the same chemical order as in the adjacent inside lattice. Dark-field (DF) TEM images are also shown, highlighting areas that correspond to twinning and matrix reflections.
Atomic-resolution high-angle annular dark-field (HAADF) scanning TEM (STEM) image recorded from the region marked by a dashed green rectangle reveals contrast indicative of segregation of heavy elements at the TB, in agreement with recent reports $[7,17]$.

The same specimen was analyzed by APT, as shown in Fig. 1(c). The Al distribution (green) is homogeneous except in the vicinity of the TB. Planar Mn segregation occurs where TBs are observed by TEM in Fig. 1(b). A composition profile across one of the TBs shows Mn enrichment from 55 at.\% in the matrix to $\sim 63$ at. $\%$ on one side of the TB, while a confined depletion down to $\sim 42.5$ at.\% $\mathrm{Mn}$ is observed on the other side. The profile is consistent with a $\mathrm{Mn}_{55} \mathrm{Al}_{45}$ composition in the untwinned and twinned regions, agreeing with previous findings $[7,13]$.

To understand the implications of the segregation, detailed $a b$ initio calculations have been performed for the (off-stoichiometric) $\tau$ phase with TBs. Since DFT is also able to resolve the small energy differences yielding the relative stability of off-stoichiometric $\mathrm{Mn}_{55.5} \mathrm{Al}_{44.5}$ composition as compared to a convex hull connecting stoichiometric MnAl and pure Mn (Supplemental Material, Fig. S4 [16]), one can have confidence in the method for this material system. We further observe that the twin boundary energies (TBEs) for both $\mathrm{Mn}_{55.5} \mathrm{Al}_{44.5}\left(0.090 \mathrm{~J} / \mathrm{m}^{2}\right)$ and $\mathrm{Mn}_{50} \mathrm{Al}_{50}$ $\left(0.118 \mathrm{~J} / \mathrm{m}^{2}\right)$ are very low. These are substantially lower than the TBEs for body-centered cubic $\mathrm{Fe}\left(0.47 \mathrm{~J} / \mathrm{m}^{2}\right.$ [18] and $\mathrm{Cr}$ $\left(0.64 \mathrm{~J} / \mathrm{m}^{2}\right)$ [19].

The calculated solution enthalpy of $\mathrm{Mn}$ for $\mathrm{Mn}_{55.5} \mathrm{Al}_{44.5}$ with respect to pure elements in the twin regions is $0.52 \mathrm{eV}$, which can be compared with the more positive value of 0.59 $\mathrm{eV}$ in the bulk region. These calculations rationalize the relative stability of the selected $\mathrm{Mn}_{55.5} \mathrm{Al}_{44.5}$ composition, the inclination of the material towards the formation of a high number density of microtwins, and the segregation of Mn observed in Fig. 1(c). They also explain general aspects of phase formation behavior in $\mathrm{MnAl}$, transcending the present study. 


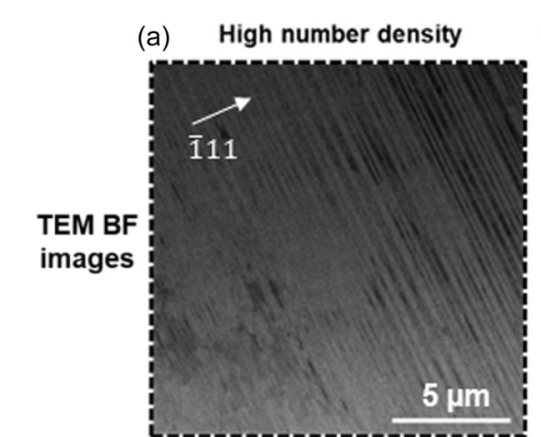

(b) Low number density

(d)

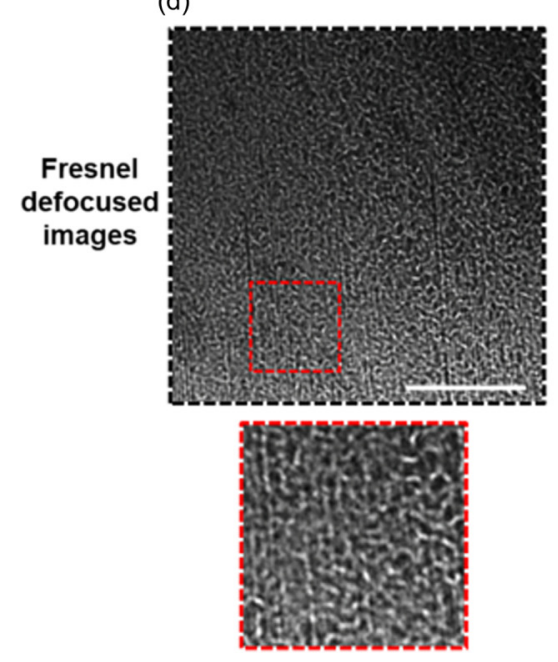

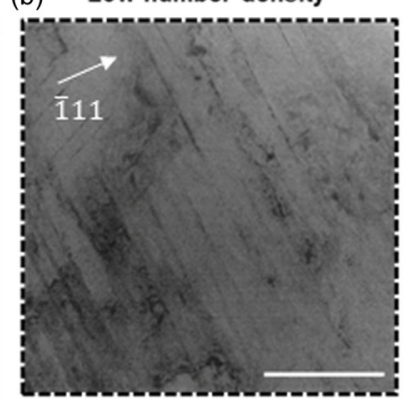

(e)
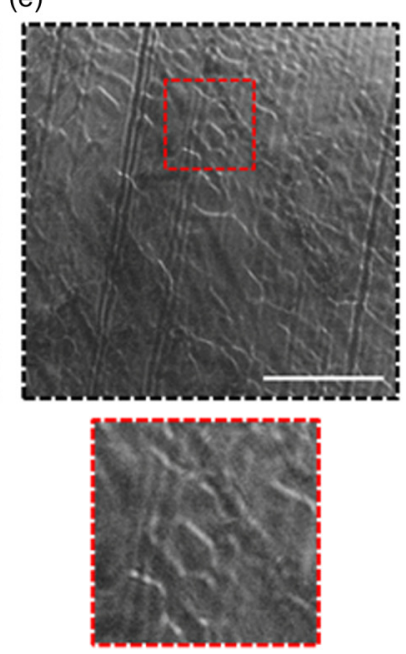

(c) a twin boundary

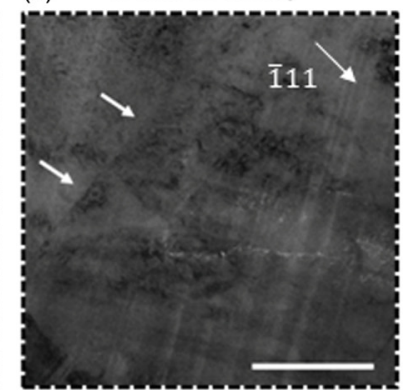

(f)

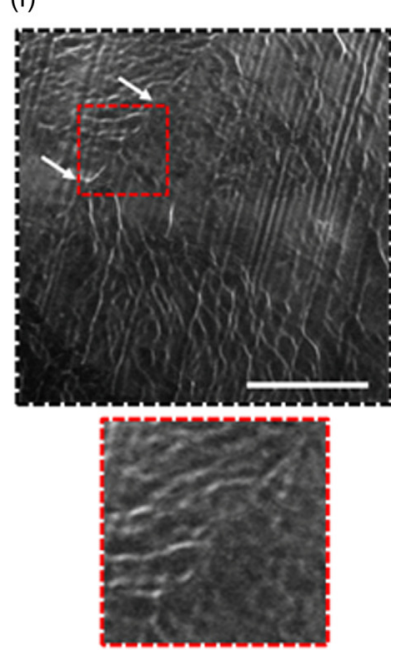

FIG. 2. Magnetic domain structure of $\tau$-MnAl. Bright-field TEM images and their corresponding Fresnel defocused images for the sample regions containing (a), (b) high and (c), (d) low number density of microtwins compared with the region containing (e), (f) no twins. The defocused values used was $0.5 \mathrm{~mm}$.

The ab initio calculations show that the excess Mn atoms are aligned antiferromagnetically to $\mathrm{Mn}$ in the sublattice of the ordered compound. At a TB, the Mn magnetic moment in the $\mathrm{Al}$ sublattice is approximately $-2.5 \mu \mathrm{B}$ and is slightly higher than the Mn magnetic moment in the Mn sublattice, which is approximately $+2.4 \mu \mathrm{B}$. Therefore, the enrichment in $\mathrm{Mn}$ leads to a reduction in local magnetization in the vicinity of TBs. This can indeed be a mechanism yielding a magnetic DW pinning [4,9]. Magnetization reversal may then occur during domain-wall propagation across microtwins, contributing to the coercivity of the alloy [5]. A similar effect of GB segregation on coercivity was recently studied in a $\mathrm{Nd}-\mathrm{Fe}-\mathrm{B}-$-based ferromagnet [20]. A reduction in ferromagnetic Fe at the GBs by the addition of $\mathrm{Nd}$ resulted in a lower magnetization of the GBs and therefore an increase in coercivity by $45 \%$ [20,21].

This mechanism alone, however, cannot explain the interplay of DWs and TBs in the (off-stoichiometric) $\tau$ phase. This becomes already apparent when performing Fresnel defocus (Lorentz) imaging in magnetic-field-free conditions to image the local magnetic domain structure at remanence (Fig. 2). Here, regions with high and low number densities of microtwins can be compared with a region that contains only one TB [Figs. 2(a)-2(c)]. The magnetic DWs appear as white and black contrast lines in the Fresnel images [Figs. 2(d)-2(f)]. The images reveal a remarkable difference in magnetic domain size as a function of microtwin density. The high twin density leads to a magnetic structure homogeneously characterized by small domain sizes. With fewer twins, the domain pattern also changes at the locations of TBs [marked with white arrows in Figs. 2(c) and 2(f)]. The observations suggest a strong influence of TBs, arising from the heat treatment, on the magnetic state. However, the consistently small domain sizes cannot only result from a pinning of moving DWs by obstacles.

Therefore, more quantitative measurements of magnetic domains and their correlation with twins were carried out using off-axis holography in the TEM (Supplemental Material S2 and S3 [16]). Figures 3(a)-3(f) show BF TEM images and corresponding maps of projected in-plane magnetic induction in regions of high and low twin density, as well as in regions without twins. Here, thin white lines in Fig. 3(b) mark the traces of TBs in Fig. 3(a). We observe the region that has a high density of twins [Fig. 3(a)], where the boundaries are 35-90 nm apart. The corresponding magnetic induction map [Fig. 3(b)] reveals a dense fishbone-shaped mosaic domain structure with rapid local changes in magnetization direction and magnetic domains that are only $20-50 \mathrm{~nm}$. These findings need to be compared with the lower density of twins. Figures 3(c)-3(f) show BF TEM images and magnetic induction maps recorded from regions with a low density of microtwins and no microtwins, respectively. They indicate a correlation of a strong decrease in the magnetic domain sizes and the increase in the twin number densities (Table I), confirming the hypothesis derived from the Fresnel images (Fig. 2). 

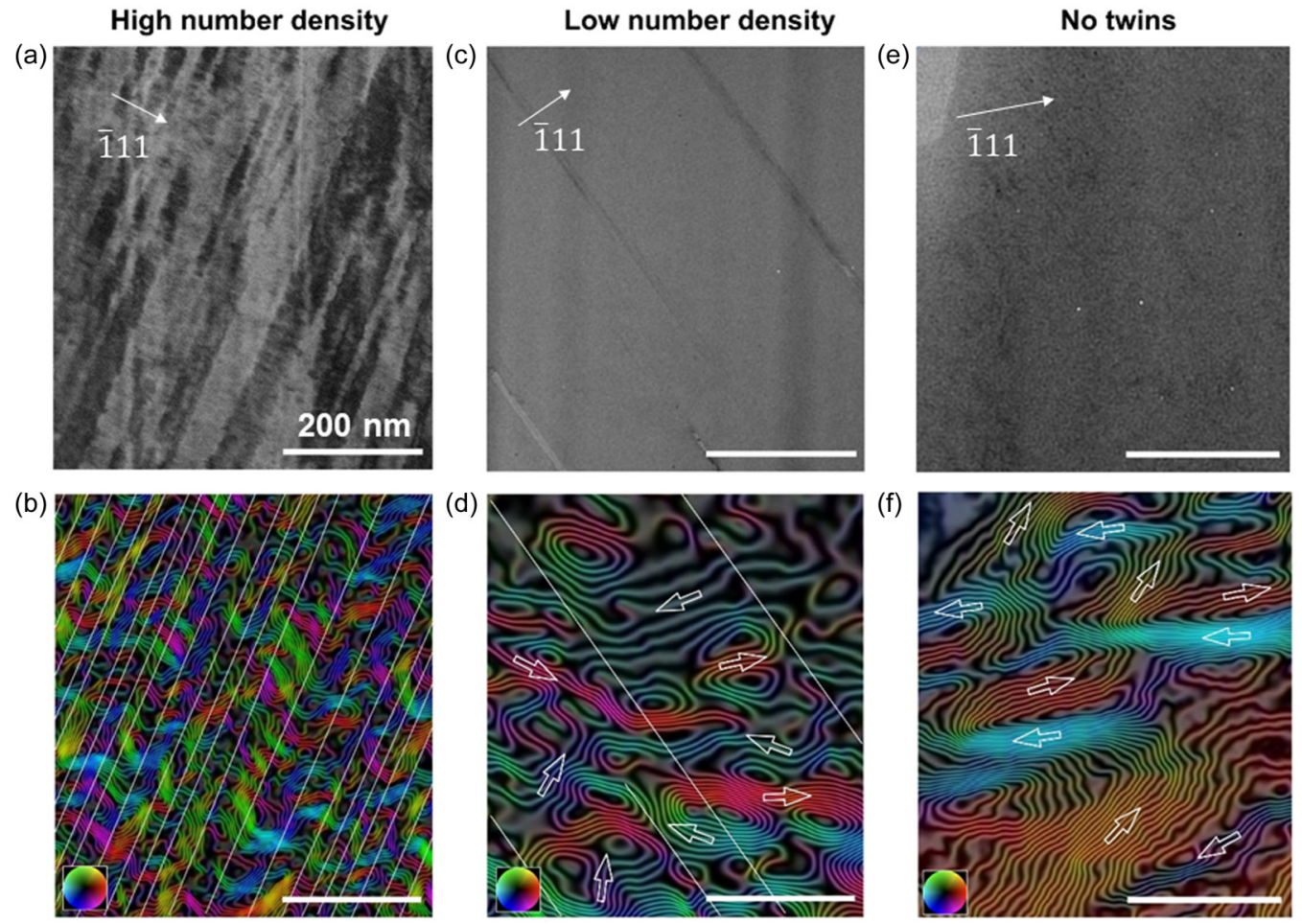

FIG. 3. Magnetic domain structure of $\tau \mathrm{MnAl}$ with higher and lower densities of microtwins. (a), (c), (e) BF TEM images and (b), (d), (f) corresponding projected in-plane magnetic induction maps recorded using off-axis electron holography. (see Supplemental Material, S3 [16]). The colors and contours indicate the direction and strength of the projected in-plane magnetic induction. The white lines in (b) and (d) mark the locations of TBs. The contour spacings are 0.15 (b) and 0.2 radians (d), (f), respectively. The scale bars are $200 \mathrm{~nm}$.

We further note that the magnetic field changes direction abruptly, forming $180^{\circ}$ DWs at the twins. Far away from the twins, the uneven distribution of magnetic field lines suggests a complex magnetic domain state, with field directions that are locally aligned out of plane. This additionally supports the assumed strong influence of TBs on the magnetic structure.

The correlation in Table I can be best understood as a consequence of a modified thermodynamic stability of the observed nanoscale domains in the presence of TBs. The results from our $a b$ initio calculations reported in Fig. 4(a) show that, in addition to phase stability, the DW energy is decreased from 0.162 to $0.010 \mathrm{~J} / \mathrm{m}^{2}$ when a TB is present, almost independent of composition. The TB therefore affects domain nucleation. For an anisotropic $L 1_{0}$-ordered alloy with its easy magnetization along the $c$ axis, a tilted $c$ axis characterizes the presence of regions that are twinned from the original ordered lattice. The calculations predict significantly lower magnetocrystalline anisotropy energy [Fig. 4(b)] and anisotropy energies (calculated between different axes) in the vicinity of a TB compared to the bulk. These results support the possibility of forming a tilted $c$ axis and hence forming a

TABLE I. Measurements of magnetic domain size as a function of twin number density.

\begin{tabular}{lccc}
\hline \hline Twin density, $\mu \mathrm{m}^{-2}$ & 14 & 125 \\
\hline Domain size, $\mathrm{nm}$ & $\geqq 200$ & $150 \pm 75$ & $35 \pm 15$ \\
\hline \hline
\end{tabular}

domain wall, i.e., overcoming intermediate spin configuration with an angle smaller than $180^{\circ}$. Within the twin structure, the easy axis is found to lie along the [11-2] twin boundary axis, influencing the domain structure. Previous studies in an $L 1_{0}$-ordered $\mathrm{FePt}(001) / \mathrm{Pt}$ thin film, using magnetic force microscopy and micromagnetic simulations, showed that the presence of microtwins can pin the domain-wall propagation $[4,9]$. However, no comments were made on the relative stability of domain walls at TBs.

In conclusion, we have demonstrated the influence of microtwins and TBs on the local magnetic domain structure in a uniaxial magnetocrystalline $L 1_{0}$-ordered $\mathrm{Mn}_{55} \mathrm{Al}_{45}$ alloy. Magnetic imaging in the TEM reveals a surprisingly fine mosaic domain structure dependent on microtwin density, as well as a strong pinning effect of domains. Such fine domains are not common in bulk hard-magnetic materials, which typically have domain sizes in the range of micrometers. Until now, the smallest domains, 100-200 nm, were observed in single-crystalline $\mathrm{Nd}-\mathrm{Fe}-\mathrm{B}$ hard magnets [22]. Here, we demonstrated that the domain size scales with the number density of twins, which can be explained by two mechanisms. First, additional Mn atoms at the TBs reduce the local magnetization, which will contribute to a DW pinning. The strength of the pinning is further evidenced by imaging the magnetic domain arrangement at remanence before and after magnetic saturation (see Supplemental Material, Fig. S5(b) [16]). A specimen with a high density of twins was applied by an in-plane field of 1.41 T. Some rearrangement of the domains was observed, but most of them did not return to their initial positions, suggesting effect on domain-wall propagation in 
(a)

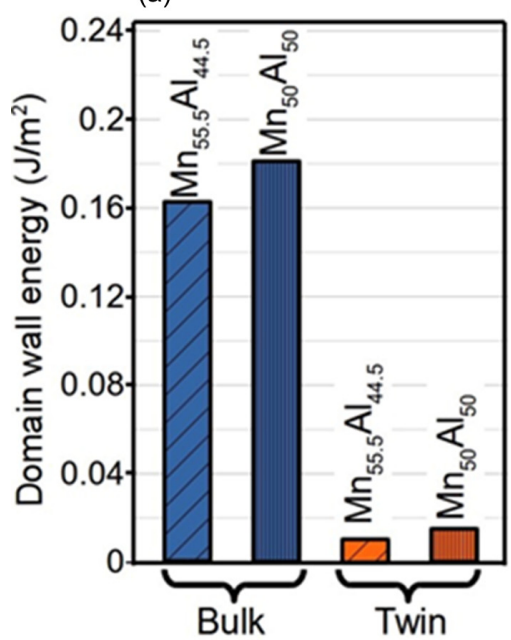

(b)

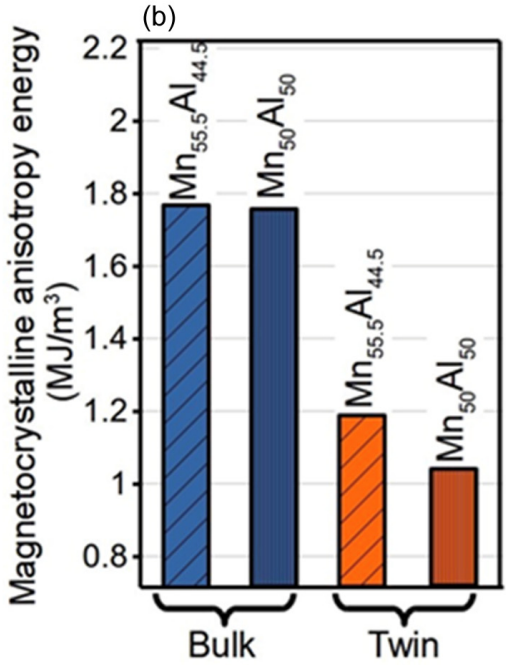

(c)
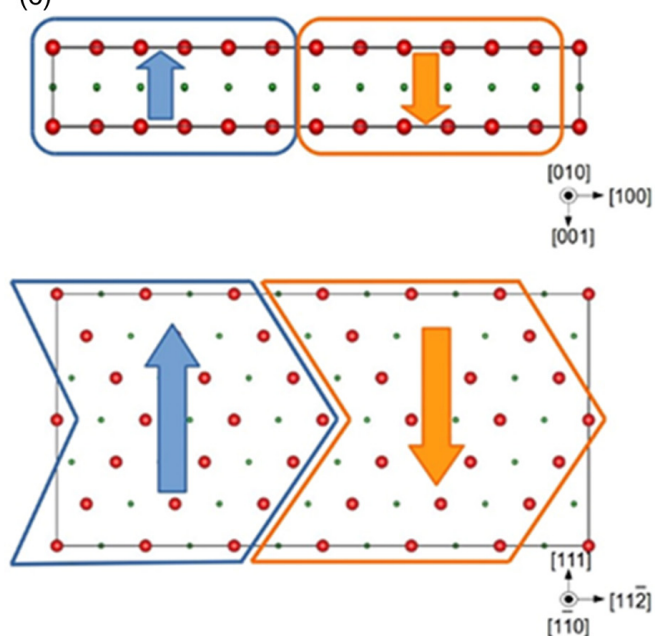

FIG. 4. Ab initio calculations on the influence of TBs on domain structure. (a) Domain-wall energies and (b) magnetocrystalline anisotropy energies in bulk and twinned regions of $\mathrm{Mn}_{55.5} \mathrm{Al}_{44.5}$ and $\mathrm{Mn}_{50} \mathrm{Al}_{50}$. (c) Bulk and TB supercells with $180^{\circ}$ domains used in the computational setup. $\mathrm{Mn}$ atoms are shown in red, while $\mathrm{Al}$ atoms are shown in green.

the presence of microtwins. Second, the ab initio calculations show that the DW energy significantly drops at the twinned boundaries and promotes easy formation of domain walls at twinned regions and hence the observed nanoscale domain structure. The direct link between the density of defects and the alloy's magnetic performance suggests approaches for the design of magnetic materials that have improved permanent magnetic properties. For instance, metallurgical thermomechanical processing can be used to increase the density of twins. As they nucleate from grain boundaries, a reduction in grain size in the original alloy will result in a higher twin density. Doping with suitable alloying additions can also further stabilize twins or modify their local magnetic properties. We have revealed which are the key metrics that can be derived from $a b$ initio calculations in order to help to design new alloys.

The authors are grateful to C. Broß, U. Tezins, and A. Sturm for technical support of the atom probe tomography and focused ion beam facilities at the Max-Planck-Institut für Eisenforschung. O.H. and T.H. acknowledge financial support by the German Research Foundation within the DFG-ANR project MAGIKID (Grant No. HI1300/13-1). This project has been in part funded by the Deutsche Forschungsgemeinschaft (DFG, German Research Foundation) - Project-ID No. 405553726 - TRR 270 and by the European Research Council (ERC) under the European Union's Horizon 2020 research and innovation programme (Grant No. 856538, Project "3D MAGiC').

D.P. generated the idea. D.P., A.K., and B.G. conceived the experiments. D.P. acquired the TEM and APT data and, with B.G., performed the analysis. A.K. performed the TEM-based magnetic characterization. O.H. and T.H. performed $a b$ initio calculations. D.R. and R.D.B. supervised the work. All authors discussed the results and contributed to their interpretation. D.P., A.K., O.H., T.H., and B.G. drafted the manuscript. All authors provided input to the manuscript.
[1] A. Hubert and R. Schäfer, Magnetic Domains: The Analysis of Magnetic Microstructures (Springer-Verlag, Berlin, Heidelberg, 1998).

[2] B. Zhang and W. A. Soffa, Magnetic domains and coercivity in polytwinned ferromagnets, Phys. Status Solidi A 131, 707 (1992).

[3] T. Klemmer, D. Hoydick, H. Okumura, B. Zhang, and W. A. Soffa, Magnetic hardening and coercivity mechanisms in L1 0 ordered FePd ferromagnets, Scr. Metall. Mater. 33, 1793 (1995).

[4] M. H. Hong, K. Hono, and M. Watanabe, Microstructure of $\mathrm{FePt} / \mathrm{Pt}$ magnetic thin films with high perpendicular coercivity, J. Appl. Phys. 84, 4403 (1998).

[5] J. P. Attané, Y. Samson, A. Marty, D. Halley, and C. Beigné, Domain wall pinning on strain relaxation defects in $\mathrm{FePt}(001) / \mathrm{Pt}$ thin films, Appl. Phys. Lett. 79, 794 (2001).

[6] D. Palanisamy, S. Singh, C. Srivastava, G. Madras, and K. Chattopadhyay, Predicting pathways for synthesis of ferromagnetic $\tau$ phase in binary Heusler alloy Al-55 Pct Mn through understanding of the kinetics of $\varepsilon-\tau$ transformation, Metall. Mater. Trans. A 47, 6555 (2016).

[7] D. Palanisamy, D. Raabe, and B. Gault, On the compositional partitioning during phase transformation in a binary ferromagnetic MnAl alloy, Acta Mater. 174, 227 (2019).

[8] J. P. Attané, D. Ravelosona, A. Marty, V. D. Nguyen, and L. Vila, Coercivity enhancement in FePt nanowires due to the suppression of available paths for domain wall propagation, Phys. Rev. B 84, 144418 (2011). 
[9] J. P. Attané, Y. Samson, A. Marty, J. C. Toussaint, G. Dubois, A. Mougin, and J. P. Jamet, Magnetic Domain Wall Propagation unto the Percolation Threshold across a Pseudorectangular Disordered Lattice, Phys. Rev. Lett. 93, 257203 (2004).

[10] H. Kōno, On the ferromagnetic phase in manganese-aluminum system, J. Phys. Soc. Jpn. 13, 1444 (1958).

[11] B. Zhang and W. A. Soffa, The structure and properties of $L 1_{0}$ ordered ferromagnets: Co-Pt, Fe-Pt, Fe-Pd and Mn-Al, Scr. Metall. Mater. 30, 683 (1994).

[12] D. Palanisamy, C. Srivastava, G. Madras, and K. Chattopadhyay, High-temperature transformation pathways for metastable ferromagnetic binary Heusler (Al-55 at.\%Mn) alloy, J. Mater. Sci. 52, 4109 (2017).

[13] Q. Zeng, I. Baker, J. B. Cui, and Z. C. Yan, Structural and magnetic properties of nanostructured $\mathrm{Mn}-\mathrm{Al}-\mathrm{C}$ magnetic materials, J. Magn. Magn. Mater. 308, 214 (2007).

[14] D. Palanisamy, G. Madras, and K. Chattopadhyay, Exploring the pathways for enhancing the hard magnetic properties of binary Al-55 at.\%Mn Heusler alloy through mechanical alloying, J. Magn. Magn. Mater. 439, 181 (2017).

[15] C. Yanar, J. M. K. Wiezorek, W. A. Soffa, and V. Radmilovic, Massive transformation and the formation of the ferromagnetic $L 1_{0}$ phase in manganese-aluminum-based alloys, Metall. Mater. Trans. A 33, 2413 (2002).
[16] See Supplemental Material at http://link.aps.org/supplemental/ 10.1103/PhysRevMaterials.5.064403 for experimental methods, magnetic imaging, and off-axis holography used in the present work.

[17] D. Palanisamy, D. Raabe, and B. Gault, Elemental segregation to twin boundaries in a MnAl ferromagnetic heusler alloy, Scr. Mater. 155, 144 (2018).

[18] Y. A. Du, L. Ismer, J. Rogal, T. Hickel, J. Neugebauer, and R. Drautz, First-principles study on the interaction of $\mathrm{h}$ interstitials with grain boundaries in $\alpha$ - and $\gamma$-Fe, Phys. Rev. B 84, 144121 (2011).

[19] H. Zheng, X.-G. Li, R. Tran, C. Chen, M. Horton, D. Winston, K. A. Persson, and S. P. Ong, Grain boundary properties of elemental metals, Acta Mater. 186, 40 (2020).

[20] J. Liu, H. Sepehri-Amin, T. Ohkubo, K. Hioki, A. Hattori, T. Schrefl, and K. Hono, Effect of Nd content on the microstructure and coercivity of hot-deformed $\mathrm{Nd}-\mathrm{Fe}-\mathrm{B}$ permanent magnets, Acta Mater. 61, 5387 (2013).

[21] T. T. Sasaki, T. Ohkubo, K. Hono, Y. Une, and M. Sagawa, Correlative multi-scale characterization of a fine grained $\mathrm{Nd}-$ Fe-B sintered magnet, Ultramicroscopy 132, 222 (2013).

[22] A. Kovács and R. E. Dunin-Borkowski, in Handbook of Magnetic Materials, edited by E. Brück, (Elsevier, Amsterdam, 2018), Vol. 27, Chap. 2, pp. 59-153. 